\title{
Does registration of serial MRI improve diagnosis of dementia?
}

\author{
Josephine Barnes $\cdot$ L. Anne Mitchell • Jonathan Kennedy • Antonio J. Bastos-Leite • \\ Suzie Barker • Manja Lehmann • R. Chris Nordstrom • Chris Frost • \\ Joseph R. Smith • Ellen Garde • Martin N. Rossor • Nick C. Fox
}

Received: 14 September 2009 /Accepted: 2 February 2010/Published online: 27 February 2010

(C) Springer-Verlag 2010

\begin{abstract}
Introduction We aimed to assess the value of a second MR scan in the radiological diagnosis of dementia.

Methods One hundred twenty subjects with clinical followup of at least 1 year with two scans were selected from a cognitive disorders clinic. Scans were reviewed as a single first scan (method A), two unregistered scans presented sideby-side $(\operatorname{method} B)$ and a registered pair $(\operatorname{method} C)$. Scans were presented to two neuroradiologists and a clinician together with approximate scan interval (if applicable) and age. Raters decided on a main and subtype diagnosis.

Results There was no evidence that differences between methods (expressed as relative odds of a correct response) differed between reviewers $(p=0.17$ for degenerative condition or not, $p=0.5$ for main diagnosis, $p=0.16$ for subtype). Accordingly, results were pooled over reviewers.
\end{abstract}

J. Barnes $(\bowtie) \cdot J$. Kennedy $\cdot$ S. Barker $\cdot$ M. Lehmann •

R. C. Nordstrom $\cdot$ C. Frost $\cdot$ J. R. Smith $\cdot$ E. Garde $\cdot$

M. N. Rossor • N. C. Fox

Dementia Research Centre, UCL Institute of Neurology, London, UK WC1N 3BG

e-mail: j.barnes@dementia.ion.ucl.ac.uk

L. A. Mitchell

Radiology Department, Austin Health,

Heidelberg, Australia

L. A. Mitchell

Radiology Department, Melbourne University,

Melbourne, Australia

\section{A. J. Bastos-Leite}

Department of Medical Imaging, Faculty of Medicine,

University of Oporto,

Oporto, Portugal
For distinguishing normal/non-progressors from degenerative conditions, the proportions correctly diagnosed were higher with methods $\mathrm{B}$ and $\mathrm{C}$ than with $\mathrm{A}$ ( $p=0.001$, both tests). The difference between method $\mathrm{B}$ and $\mathrm{C}$ was not statistically significant $(p=0.18)$. For main diagnosis, the proportion of correct diagnoses were highest with method C for all three reviewers; however, this was not statistically significant comparing with method A $(p=0.23)$ or with method $\mathrm{B}(p=0.16)$. For subtype diagnosis, there was some evidence that method $\mathrm{C}$ was better than method $\mathrm{A}(p=0.01)$ and $\mathrm{B}(p=0.048)$.

Conclusions Serial MRI and registration may improve visual diagnosis in dementia.

Keywords Registration · Serial MRI · Dementia .

Diagnosis $\cdot$ Visual assessment

\section{A. J. Bastos-Leite}

Lysholm Department of Neuroradiology,

National Hospital for Neurology and Neurosurgery,

Queen Square,

London, UK

C. Frost

Department of Medical Statistics,

London School of Hygiene and Tropical Medicine,

London, UK

E. Garde

Danish Research Centre for Magnetic Resonance,

Copenhagen University Hospital Hvidovre,

Hvidovre, Denmark DK-2650 


\section{Introduction}

Dementia is one of the leading global health and socioeconomic problems [1]. Many diseases can cause dementia, but Alzheimer's disease (AD) is the most common [2, 3]. Vascular dementia and dementia with Lewy bodies (DLB) are the next most common $[2,3]$ whilst frontotemporal lobar degeneration (FTLD) is the third most common dementing disease under 65 years [4].

In dementia, a definitive diagnosis requires histological examination of brain tissue, and clinical diagnosis may be difficult, particularly in the early stages. The hallmarks of $\mathrm{AD}$ are intracellular neurofibrillary tangles, extracellular amyloid plaques and cerebral atrophy, which increase with disease severity [5]. Clinically, AD typically presents with amnestic problems, but there are a range of differing presentations including posterior and anterior variants [6] when subjects can present with atypical symptoms such as visual problems and behavioural disturbances, respectively. DLB can occur in a pure form or can co-exist with $\mathrm{AD}$ pathology, and similarly mixed $\mathrm{AD} /$ vascular pathologies are seen at autopsy in approximately $30 \%$ of clinically diagnosed AD cases [2]. FTLD may be caused by several different neurodegenerative pathologies most commonly with tau- or ubiquitin-positive neuronal inclusions [7]. According to clinical presentation, FTLD can be subdivided into language variants such as semantic dementia (SD) and progressive non-fluent aphasia (PNFA) and behavioural variant FTLD (bvFTD) [8].

Whilst definitive diagnosis of the neurodegenerative dementias requires histology, the characteristic pattern of neuronal loss is reflected in the pattern of regional brain atrophy. There is thus a great interest in using imaging to improve or support clinical diagnoses. Recently, medial temporal lobe atrophy has been included in new proposed diagnostic criteria for $\mathrm{AD}$ [9], whilst focal frontal and/or temporal atrophy are part of diagnostic consensus criteria for FTLD [10]. The different clinical presentations of FTLD are associated with different patterns of atrophy; SD patients typically having predominant often asymmetric temporal lobe atrophy; PNFA having (left) perisylvian atrophy whilst preferential frontal atrophy is seen in bvFTD. Despite these characteristic associations, there is some overlap and some dissociation in a number of cases.

Visual inspection of the pattern of atrophy on MRI is probably the most common imaging assessment used to diagnose specific diseases causing dementia in clinical practice. Usually, a single scan is performed, but in some cases, a further scan is taken to assess progression and clarify diagnosis. Positional matching (registration) of scans has been a valuable research tool in understanding the natural history of neurodegenerative diseases [11]. Registration allows different analyses: visual analysis and assessment of progression, calculation of atrophy [12, 13], estimation of atrophy location $[14,15]$ and estimation of volume changes in brain sub-regions [16]. Increased atrophy rates of whole brain and sub-regions are predictive of clinical progression to a dementia diagnosis [17]. However, the value of serial imaging and registration in a clinical setting is not well understood.

We aimed to assess added diagnostic value of performing a second scan and whether registration of the second scan to baseline would improve diagnostic accuracy further. Visual diagnosis was compared with (a) clinical diagnosis and (b) pathological confirmation where available.

\section{Materials and methods}

\section{Subject selection}

This retrospective study was conducted using subjects referred to the specialist cognitive disorders clinic at the National Hospital for Neurology and Neurosurgery, who had more than one MRI scan, had attended clinic more than twice and remained in follow-up for 12 months or more. Three hundred and one subjects with at least two MRI scans were identified. In addition, subjects known to have either pathological or genetic confirmation of disease and two scans were included.

Clinical notes were reviewed in order to categorise the subjects according to their final diagnosis (with final diagnosis being either the last clinical or pathological diagnosis). Categories included those with a non-progressive or degenerative condition and normals and those with progressive degenerative diseases: AD [18], FTLD [10] or other. Subjects were excluded if a diagnosis was not stated. This resulted in 120 subjects with two usable MRI scans and a clinical, genetic or pathological diagnosis. Some of the subjects have been included in different studies [19-21].

Each subject was given an identification number, and this number replaced subject names on all scans. The date of the scan remained unedited. Baseline and follow-up images were co-registered using 12 degrees of freedom [22] and differential bias correction [23] to reduce intensity nonuniformity differences.

\section{Presentation of scans}

Scans were reviewed as a single first scan (method A), the two unregistered presented side-by-side $(\operatorname{method} \mathrm{B})$ and the registered pair (method $\mathrm{C}$ ). In order to minimise bias, a design based on the three-period cross-over design [24, 25] was utilised to determine the order in which scans were presented to the reviewers. Stratified by final diagnosis, subjects were randomised into six groups of 20 subjects. 
Reviewers were presented with scans in 18 blocks each including results from a single group using a particular methodology (A, B or C). The six groups were distinguished by the order in which the methodologies were reviewed ( $\mathrm{ABC}, \mathrm{ACB}, \mathrm{BAC}, \mathrm{BCA}, \mathrm{CAB}$ or $\mathrm{CBA})$. $\mathrm{A}$ second randomisation was used to order the subjects within each group (this order remained the same for each methodology). Finally, the ordering of the groups was determined using a restricted randomisation that ensured that the first three groups selected made up a Latin Square, as did the final three groups. The selected ordering was "CBA", "ACB", "BAC", "ABC", "CAB" and "BCA". Hence, the reviewer was first presented with 20 registered scan pairs (method C) from the "CBA" group, then with 20 single scans (method A) from the "ACB" group and so on.

Subjects and methods were viewed independently by a clinician (neurologist in training with 2 years experience at the Dementia Research Centre) and two neuroradiologists. Only the approximate scan interval (if method B or C) and age at first scan were given. The reviewer had to decide on a main and specific (subtype) diagnosis. Main diagnoses included: AD, FTLD, normal, non-specific and other. Subtype diagnoses were typical and posterior variant for $\mathrm{AD}$ and behavioural variant (bv), temporal variant (tv) and PNFA for FTLD. A certainty rating of diagnosis: low, medium or high, was also made. If two scans were presented, the reviewer had to decide whether progressive pathological atrophy was present. The start and end time of each 20 scan session was recorded to estimate assessment time by method. Once all scans were reviewed, the first 20 subjects were reviewed again in the same order using each method to provide a measure of within-rater repeatability.

\section{Data analysis}

Prior to analysis, the diagnostic categories of non-specific and other were collapsed since specific diagnoses were rarely recorded when "other" was used. Data analysis was performed using STATA version 10 (STATA Corporation, College Station, TX, USA) and SAS (SAS Institute Inc., Cary, NC, USA).
In the primary analysis, the (binary) outcome was whether the four category main diagnosis (normal/non-progressor, $\mathrm{AD}$, FTLD or other pathology) was correct. A secondary analysis merely considered whether the broad categorisation of "normal/non-progressor" vs "degenerative condition" (AD, FTLD and other pathology combined) was correct. In a further secondary analysis restricted to those subjects with $\mathrm{AD}$ and FTLD, the (binary) outcome was whether or not the subtype diagnosis was correct. Since the study used a crossover design, conditional logistic regression was used to account for subject-specific effects. As is standard in crossover trials [24, 25], all analyses were adjusted for period. For all of these outcomes, predictor variables were (1) method, (2) reviewer and (3) interaction of (1) and (2). A weighted Kappa statistic was used to assess within-rater repeatability with a main diagnosis disagreement between a normal/nonprogressive diagnosis and a neurodegenerative disease (i.e. normal vs FTLD) weighted at zero, and within degenerative disease disagreement (i.e. AD vs FTLD) weighted as 0.75 . For subtype analyses, disagreements across AD and FTLD subtypes were weighted at zero, and the within AD or FTLD disagreements (i.e. posterior vs typical for $\mathrm{AD}$ ) were weighted at 0.75 .

As a check on the reliability of the findings, we investigated whether there was evidence of a difference in results according to pathological disease diagnosis. This was performed by including interactions between method and a "pathological" indicator.

A further set of analyses considered certainty of diagnosis (three levels) as an ordered categorical response. For each reviewer, a proportional odds model with subject as a random effect [24, pp 311-313] was used to relate this outcome variable to method, correctness of diagnosis and their interaction (with adjustment for period effects).

\section{Results}

Details of subjects are reported in Tables 1 and 2. Those within the category of "non-progressor" included: three with static cognitive impairment, one monophasic illness

Table 1 Subject demographics as main diagnosis.

\begin{tabular}{llll}
\hline & Normals and non-progressors & AD & FTLD \\
\hline$N$ (no with established diagnosis $^{\mathrm{a}}$ ) & $18(4)$ & $56(21)$ & $27(16)$ \\
Male/female & $12 / 6$ & $28 / 28$ & $19(10)$ \\
Mean age/years (SD) at baseline scan & $58(13)$ & $63(9)$ & $17 / 10$ \\
Mean scan interval/months (SD) & $19(10)$ & $16(10)$ & $61(7)$ \\
\hline
\end{tabular}

${ }^{\text {a }}$ Established describes those subjects for whom either genetic or pathological proof of disease is available. Four normal controls include one with a normal post mortem, two considered to be normal/depressed and one with a positive genetic test for pre-senilin 1 who remained asymptomatic for at least 5 years 
Table 2 Subject demographics by subtype diagnosis.

$b v$ behavioural variant, $t v$ temporal variant, $P N F A$ progressive non-fluent aphasia

${ }^{\text {a }}$ Typical describes AD subjects with no mention of posterior cortical atrophy/biparietal $\mathrm{AD}$

\begin{tabular}{|c|c|c|c|c|c|}
\hline & \multicolumn{2}{|l|}{$\mathrm{AD}$} & \multicolumn{3}{|l|}{ FTLD } \\
\hline & Typical $^{\mathrm{a}}$ & Posterior & bv & tv & PNFA \\
\hline $\mathrm{N}$ & 43 & 13 & 13 & 11 & 3 \\
\hline Mean age/years (SD) at baseline scan & $63(10)$ & $62(8)$ & $60(9)$ & $62(6)$ & $65(5)$ \\
\hline Mean scan interval/months (SD) & $17(10)$ & $12(5)$ & $17(14)$ & $22(12)$ & $13(5)$ \\
\hline
\end{tabular}

affecting frontal lobes, two with depression, one with vestibular dysfunction, one with a resolved monophasic neuropsychiatric illness of unknown cause, one sleep apnoea, one pre-senilin 1 carrier who remained asymptomatic 5 years after the repeat scan, one with subjective memory impairment and one with temporal lobe epilepsy. Those within the "other" category included: one multisystem atrophy, three DLB, one Niemann-Pick disease type $\mathrm{C}$, six vascular dementia, one Parkinson's disease, one CADASIL, one subject with a frontal and temporal presentation with atypical FTLD pathology and clinical features, two prion disease, one with mixed vascular and temporal lobe epilepsy and two with progressive supranuclear palsy. Two subjects in the AD category had mixed vascular dementia and AD.

\section{Comparison of inter-reviewer patterns}

Table 3 shows the proportions of correct diagnoses for each reviewer using each of the three methods. For each outcome (degenerative condition or not, main diagnosis, subtype diagnosis), the odds of a correct response differed between the three reviewers. However, there was no evidence that the differences between methods (expressed as a relative odds of a correct response) differed between reviewers ( $p=0.17$ for degenerative condition or not, $p=0.5$ for main diagnosis, $p=0.16$ for subtype). Accordingly, we primarily report results from a pooled analysis, adjusting for main effects of reviewer, period and their interaction.

For distinguishing normal/non-progressors from degenerative conditions, the proportions correctly diagnosed were higher with methods $\mathrm{B}$ and $\mathrm{C}$ than with $\mathrm{A}$ for all three reviewers. Pooling over reviewers, the relative odds of a correct response with method $\mathrm{C}$ compared with A was 4.3 (95\% CI 2.2, 8.5, $p<0.001)$. The analogous odds ratio comparing method B with A was 2.8 (95\% CI 1.5, 5.2, $p=$ 0.001). The difference between method B and $C$ was not statistically significant $(p=0.18)$. Although the global test of interaction between reviewer and method was not statistically significant, there were some quite large differences between reviewers. In particular, for the clinician and neuroradiologist 1 , performance with $\mathrm{B}$ and $\mathrm{C}$ was almost identical, whilst for neuroradiologist 2, performance was better with $\mathrm{C}$.
For main diagnosis, the proportion of correct diagnoses was highest with method $\mathrm{C}$ for all three reviewers. However, neither the comparison with method A (odds ratio $=1.3,95 \%$ CI $0.8,2.0, p=0.23$ ) or that with $\mathrm{B}$ (odds ratio $=1.4,95 \%$ CI $0.9,2.1, p=0.16$ ) achieved statistical significance. For the clinician, the percentage agreement and weighted Kappa statistic for reliability for main diagnosis were $73.75 \%$ and 0.38 for method A, $92.50 \%$ and 0.75 for method $\mathrm{B}$ and $80.00 \%$ and 0.36 for method $\mathrm{C}$, respectively. For neuroradiologist 1 , the analogous statistics were $90.00 \%$ and 0.64 for method A, $90.00 \%$ and 0.62 for method B and $92.50 \%$ and 0.64 for method C. For neuroradiologist 2 , the analogous statistics were $100 \%$ and 1.00 for method A, 95.00\% and 0.84 for method B and $76.25 \%$ and 0.31 for method C.

For subtype diagnosis, pooling over reviewers, there was some evidence that method $\mathrm{C}$ was better than method $\mathrm{A}$ (odds ratio $=1.9,95 \%$ CI 1.2, 3.1, $p=0.01$ ) and $\mathrm{B}$ (odds ratio $=1.6,95 \%$ CI 1.0, 2.7, $p=0.048$ ). Although the global test of interaction between reviewer and method was nonstatistically significant, there were some quite large differences between reviewers. Most strikingly, the clinician's correct diagnosis rate was the same with method A as with $\mathrm{C}$, whereas for neuroradiologist 1 their rate was substantially lower. For the clinician, the percentage agreement and weighted Kappa statistic for reliability for subtype were $90.38 \%$ and 0.41 for method A, 96.67\% and 0.90 for method B and $98.33 \%$ and 0.95 for method C. Analogous statistics for neuroradiologist 1 were $95.00 \%$ and 0.90 for method A, $97.92 \%$ and 0.95 for method B and $87.50 \%$ and 0.71 for method C. For neuroradiologist 2, the analogous statistics were $97.06 \%$ and 0.93 for method A, $98.44 \%$ and 0.97 for method B and $84.62 \%$ and 0.64 for method C.

\section{Pathological/genetic proof of disease}

For conditional logistic regression analyses where the outcome was a correct main diagnosis or subtype diagnosis, we found no evidence that the differences between the methods (expressed as odds ratios) depended upon whether or not there was pathological confirmation of diagnosis and main diagnosis ( $p>0.5$ for both outcomes, joint test of interactions between method and a pathology-proven indicator). For distinguishing normal/non-progressors from 
degenerative conditions, there was evidence $(p=0.01$, joint test of interactions) that the odds ratios favouring methods $\mathrm{B}$ and $\mathrm{C}$ over $\mathrm{A}$ were larger when pathology was proven than when unproven.

\section{Certainty of diagnosis}

The distribution of diagnostic certainty for both correct and incorrect main diagnoses in all 120 subjects is shown in Fig. 1.

For the clinician, when a correct diagnosis was made, there was borderline statistically significant evidence of variation in certainty according to method $(p=0.07$, joint test). Specifically, a difference in certainty was seen for method B vs method A with an increase in the proportion of medium and highly certain diagnoses $(p=0.03)$. An analogous pair-wise comparison of method $\mathrm{C}$ with method A was of borderline statistical significance $(p=0.09)$. Further, when the diagnosis was wrong, there was significant variation in certainty according to method with an increase in proportion of high certainty from methods $\mathrm{A}$ to $\mathrm{C}(p=0.03)$. Generally, when a correct diagnosis was made, the certainty was higher than when an incorrect diagnosis was made.

When neuroradiologist 1 was correct, there was significant variation in certainty by method $(p=0.03$, joint test). However, when wrong, there was no evidence in variation of certainty by method. When correct, a difference in pattern was seen for method C vs method A with an increase in the proportion of medium and highly certain diagnoses $(p=0.01)$. An analogous pair-wise analysis for method B vs method A was of borderline statistical significance $(p=0.09)$. Whether this neuroradiologist was correct or incorrect with method A had little impact on certainty; however, using both methods B and C when a correct diagnosis was made, the certainty was also greater.

When neuroradiologist 2 was correct, there was significant variation in certainty by method ( $p=0.03$, joint test). Certainty was greater with method $\mathrm{C}$ than with either of the other methods. The same trend was significant $(p=0.04$, joint test) when the neuroradiologist was incorrect. Generally, when a correct diagnosis was made, the certainty was higher than when an incorrect diagnosis was made.

\section{Timings}

Table 4 shows an estimate of the assessment time in seconds each reviewer needed to view a subject according to method. For all reviewers, method B took longest, and method A took shortest to view. Neuroradiologist 2 took most time using all methods, and neuroradiologist 1 took least time using all methods. 
Fig. 1 Distribution of diagnostic certainty according to correctness and reviewer

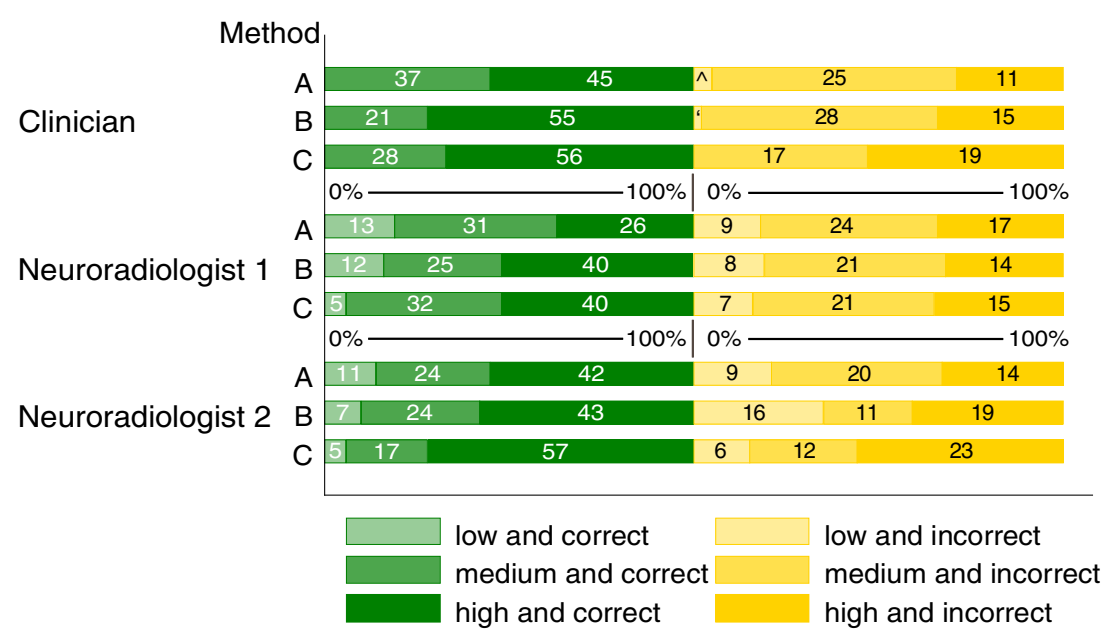

Low, medium and high relates to level of certainty which is expressed as a percentage of total correct and incorrect answers. Correct and incorrect relates to whether the main diagnosis was correct. Numerators are shown over the colour bars apart from: ${ }^{\wedge}=2, "=1$.

\section{Discussion}

This study assessed the relative value of visual assessment of single time-point MRI and serial MRI in the radiological diagnosis of dementia. Visual assessments were compared with clinical diagnosis or with pathological diagnosis when available. We found that serial imaging was useful in determining whether a subject had a degenerative condition, with incorrect classification occurring in $16-23 \%$ of cases with single scans, which lowered to $8-22 \%$ using serial imaging. However, specific identification of main diagnosis was incorrect $30-42 \%$ of the time with no statistically significant advantage provided by serial imaging. Where main diagnoses were AD and FTLD, there was some evidence that serial imaging and, in particular, registration improved subtype diagnosis for the reviewers. Although non-significant, there were some differences between reviewers in terms of their pattern of correct subtype diagnoses according to method. For neuroradiologist 1, in particular, serial imaging and additionally registration of the serial images increased the certainty of diagnosis when this diagnosis was correct. For all reviewers assessing serial images, unregistered images took longer

Table 4 Mean estimate of the amount of time in seconds each subject was reviewed according to method and reviewer (mean represents total time taken over six viewing sessions per method/120).

Clinician Neuroradiologist 1 Neuroradiologist 2

\begin{tabular}{lrrr}
\hline Method A, single & 94 & 59 & 103 \\
$\begin{array}{l}\text { Method B, serial } \\
\text { unregistered }\end{array}$ & 138 & 110 & 155 \\
$\begin{array}{c}\text { Method C, serial } \\
\text { registered }\end{array}$ & 112 & 80 & 140 \\
\hline
\end{tabular}

than registered, and both serial scan presentations were more time consuming than the single baseline scan.

Registration is an automated procedure which often requires little operator intervention; in this particular study, brain to brain registration was performed using an automated registration technique [22]. This involved semiautomated segmentation of the brain regions prior to registration [26] taking approximately $10 \mathrm{~min}$ per scan. Such processing may be unnecessary to achieve registration of sufficient quality for reviewing serial images. Many algorithms exist which enable registration to be performed $[12,27-30]$, and these are often part of packages which have a graphical user interface to aid non-technical staff. In the future, it is possible that such techniques will be applied in real time, whilst subjects are in the scanner. This will not only provide a neuroradiologist with information at the time of reporting but will also allow quality evaluation of serial images.

Other studies have assessed the use of different techniques in diagnosing subjects or predicting group membership. Visual rating such as medial temporal atrophy ratings scales [31] has been shown to distinguish clinically diagnosed $\mathrm{AD}$ patients from controls with a sensitivity of approximately $85 \%$ for a specificity of $80 \%$ [32]. Width of temporal lobe on MRI has been shown to have a sensitivity of $86 \%$ and a specificity of $93 \%$ when discriminating AD subjects from controls [33]. Pair-wise differences between controls and $\mathrm{AD}, \mathrm{SD}$ and bvFTD patient groups using visual ratings of multiple regions have also been shown [34]. Volumetric analysis has been shown to discriminate between controls and $\mathrm{AD}$ subjects [35] and predict the subsequent decline of those only mildly affected [17]. Differences in atrophy patterns have been shown between AD and FTLD (semantic dementia) subjects with those with semantic 
dementia having a more asymmetric (right volume $>$ left volume) and more anterior presentation than $\operatorname{AD}[36,37]$. More complex techniques such as cortical thickness analysis have also been shown to differentiate AD subjects from controls [38].

The strengths of this study are that the images were obtained retrospectively from real clinical data of varying quality with a variety of clinical diagnoses which mimics a genuine clinical situation. The size of this serial imaging dataset was large containing many subjects with proven diagnoses. The design utilised principles from that of crossover trials to ensure that comparisons between reviewers using different methods on the same subjects could be made without introducing bias. This included ensuring that reviewers had a "passive wash-out period" which was accomplished by ensuring that the same subject twice was not reviewed twice on the same day by the same reviewer, so that diagnosis for a given subject was less likely to be remembered.

This study has a number of limitations. First, the equipoise of subjects is different to many clinical settings; subjects referred to our tertiary referral centre are often those who are difficult to diagnose or may have relatively rare conditions. In addition, we selected subjects with pathologically or genetically confirmed diseases to increase the certainty of diagnosis. Subjects with a post mortem or brain biopsy are often those difficult to diagnose clinically. In addition, in those subjects without disease confirmation, the diagnosis was reliant on clinical notes which may have omitted certain diagnostically relevant information. As a result, we had a small number of cases which were classified as "non-progressor" who may have had progressive disease which was considered to be static in the medical notes or a subsequent diagnosis of a progressive condition had not yet been reached. Equally, those diagnosed with a progressive condition such as AD may have been misdiagnosed. We also excluded subjects for which there was an unclear diagnosis in the clinical notes with no pathological confirmation. Although the aim of this experiment was to compare methods (i.e. a paired design), the absolute levels of accuracy of diagnosis may be inflated as a result of this exclusion.

The quality of the imaging used in this study was not always high with some of the subjects' scans having artefacts such as movement. Although we selected pairs of scans performed on the same scanner, we did not control for acquisition which in some cases changed over the scanning interval. Differing scanners and acquisitions may have affected the ability of the raters to determine diagnosis. Ideally, in either a real clinical situation or a study, consistent scanning acquisitions would be used. The inter-scan interval also varied greatly within all groups. Although we excluded subjects with scanning intervals of less than 4 months and found no evidence of an effect of interval on main diagnosis, it may be that progressive atrophic patterns are difficult to assess at shorter intervals. We only assessed T1-weighted imaging, and for the assessment of vascular lesions, T2weighted imaging would be more useful. Serial scanning in vascular dementia may not be necessary since a diagnosis can often be made with a single scan.

We only have results from three raters (a clinician and two neuroradiologists), which limits our ability to generalise from our findings. A greater number of reviewers would enable a more robust analysis of the influence of clinical experience on the accuracy of diagnosis by method. However, it is possible that the non-significant differences between them could be explained by their different training and expertise: The clinician is used to distinguishing subtypes of AD and FTLD, neuroradiologist 1 is used to comparing images side-by-side and neuroradiologist 2 has experience of all methods. In particular, it may be that registration improved performance of the neuroradiologists with subtype diagnosis as different patterns of atrophy appeared more distinct. However, more neuroradiologists and clinicians would need to review the dataset in order to properly assess these hypotheses. Finally, although our study was carefully designed to mimic "real life" diagnosis, it is a retrospective study. A prospective study, perhaps using designs such as that adopted to assess the merits of different screening regimes in breast cancer screening [39], is also needed to substantiate our results in a more controlled setting. Such a study could also incorporate clinical histories of patients which were not provided to the raters in our retrospective study. In addition, the added diagnostic value of quantitative MR measures, such as global and regional brain volumes and losses over time, needs to be evaluated.

\section{Conclusions}

Serial scanning and registration may be of benefit in the differential diagnosis of dementia. The extent of the benefits may depend on the experience of the professional making the diagnosis.

Acknowledgements This work was undertaken at the University College London Hospital/University College London, which received a proportion of funding from the UK Department of Health's National Institute for Health Research Biomedical Research Centres funding scheme. The Dementia Research Centre is an Alzheimer's Research Trust Coordinating Centre. J Barnes is supported by an Alzheimer's Research Trust (UK) Research Fellowship with the support from the Kirby Laing Foundation, M Lehmann holds an Alzheimer's Society PhD Scholarship, N Fox is supported by a Medical Research Council (MRC, UK) Senior Clinical Fellowship and M Rossor and N Fox are National Institute for Health Research senior investigators.

Conflict of interest statement We declare that we have no conflict of interest. 


\section{References}

1. Ferri CP, Prince M, Brayne C, Brodaty H, Fratiglioni L, Ganguli M, Hall K, Hasegawa K, Hendrie H, Huang Y, Jorm A, Mathers C, Menezes PR, Rimmer E, Scazufca M (2005) Global prevalence of dementia: a Delphi consensus study. Lancet 366:2112-2117

2. Jellinger KA (2006) Clinicopathological analysis of dementia disorders in the elderly - an update. J Alzheimers Dis 9:61-70

3. Barker WW, Luis CA, Kashuba A, Luis M, Harwood DG, Loewenstein D, Waters C, Jimison P, Shepherd E, Sevush S, Graff-Radford N, Newland D, Todd M, Miller B, Gold M, Heilman K, Doty L, Goodman I, Robinson B, Pearl G, Dickson D, Duara R (2002) Relative frequencies of Alzheimer disease, Lewy body, vascular and frontotemporal dementia, and hippocampal sclerosis in the State of Florida Brain Bank. Alzheimer Dis Assoc Disord 16:203-212

4. Ratnavalli E, Brayne C, Dawson K, Hodges JR (2002) The prevalence of frontotemporal dementia. Neurol 58:1615-1621

5. Braak H, Braak E (1991) Neuropathological staging of Alzheimer-related changes. Acta Neuropathol 82:239-259

6. Galton CJ, Patterson K, Xuereb JH, Hodges JR (2000) Atypical and typical presentations of Alzheimer's disease: a clinical, neuropsychological, neuroimaging and pathological study of 13 cases. Brain 123:484-498

7. Mackenzie IRA, Rademakers R (2007) The molecular genetics and neuropathology of frontotemporal lobar degeneration: recent developments. Neurogenetics 8:237-248

8. Neary D, Snowden J, Mann D (2005) Frontotemporal dementia. Lancet Neurol 4:771-780

9. Dubois B, Feldman HH, Jacova C, DeKosky ST, BarbergerGateau P, Cummings J, Delacourte A, Galasko D, Gauthier S, Jicha G, Meguro K, O’Brien J, Pasquier F, Robert P, Rossor M, Salloway S, Stern Y, Visser PJ, Scheltens P (2007) Research criteria for the diagnosis of Alzheimer's disease: revising the NINCDS-ADRDA criteria. Lancet Neurol 6:734-746

10. Neary D, Snowden JS, Gustafson L, Passant U, Stuss D, Black S, Freedman M, Kertesz A, Robert PH, Albert M, Boone K, Miller BL, Cummings J, Benson DF (1998) Frontotemporal lobar degeneration: a consensus on clinical diagnostic criteria. Neurol 51:1546-1554

11. Fox NC, Freeborough PA (1997) Brain atrophy progression measured from registered serial MRI: validation and application to Alzheimer's disease. J Magn Reson Imaging 7:1069-1075

12. Smith SM, Zhang YY, Jenkinson M, Chen J, Matthews PM, Federico A, De Stefano N (2002) Accurate, robust, and automated longitudinal and cross-sectional brain change analysis. Neuroimage 17:479-489

13. Freeborough PA, Fox NC (1997) The boundary shift integral: an accurate and robust measure of cerebral volume changes from registered repeat MRI. IEEE Trans Med Imaging 16:623-629

14. Fox NC, Crum WR, Scahill RI, Stevens JM, Janssen JC, Rossor MN (2001) Imaging of onset and progression of Alzheimer's disease with voxel-compression mapping of serial magnetic resonance images. Lancet 358:201-205

15. Scahill RI, Schott JM, Stevens JM, Rossor MN, Fox NC (2002) Mapping the evolution of regional atrophy in Alzheimer's disease: unbiased analysis of fluid-registered serial MRI. Proc Nat Acad Sci (USA) 99:4703-4707

16. Barnes J, Scahill RI, Boyes RG, Frost C, Lewis EB, Rossor CL, Rossor MN, Fox NC (2004) Differentiating AD from aging using semiautomated measurement of hippocampal atrophy rates. Neuroimage 23:574-581

17. Jack CR Jr, Shiung MM, Weigand SD, O’Brien PC, Gunter JL, Boeve BF, Knopman DS, Smith GE, Ivnik RJ, Tangalos EG, Petersen RC (2005) Brain atrophy rates predict subsequent clinical conversion in normal elderly and amnestic MCI. Neurol 65:1227-1231
18. McKhann G, Drachman D, Folstein M, Katzman R, Price D, Stadlan EM (1984) Clinical diagnosis of Alzheimer's disease: report of the NINCDS-ADRDA work group under the auspices of Department of Health and Human Services Task Force on Alzheimer's Disease. Neurol 34:939-944

19. Barnes J, Godbolt AK, Frost C, Boyes RG, Jones BF, Scahill RI, Rossor MN, Fox NC (2007) Atrophy rates of the cingulate gyrus and hippocampus in $\mathrm{AD}$ and FTLD. Neurobiol Aging 28:20-28

20. Kloppel S, Stonnington CM, Barnes J, Chen F, Chu C, Good CD, Mader I, Mitchell LA, Patel AC, Roberts CC, Fox NC, Jack CR Jr, Ashburner J, Frackowiak RS (2008) Accuracy of dementia diagnosis: a direct comparison between radiologists and a computerized method. Brain 131:2969-2974

21. Kloppel S, Stonnington CM, Chu C, Draganski B, Scahill RI, Rohrer JD, Fox NC, Jack CR Jr, Ashburner J, Frackowiak RS (2008) Automatic classification of MR scans in Alzheimer's disease. Brain 131:681-689

22. Woods RP, Grafton ST, Holmes CJ, Cherry SR, Mazziotta JC (1998) Automated image registration: I. General methods and intrasubject, intramodality validation. J Comput Assist Tomogr 22:139-152

23. Lewis EB, Fox NC (2004) Correction of differential intensity inhomogeneity in longitudinal MR images. Neuroimage 23:75-83

24. Jones B, Kenward MG (2003) Design and analysis of cross-over trials, 2nd edn. Chapman and Hall, London

25. Senn S (2002) Cross-over trials in clinical research, 2nd edn. Wiley, Chichester

26. Freeborough PA, Fox NC, Kitney RI (1997) Interactive algorithms for the segmentation and quantitation of 3-D MRI brain scans. Comput Methods Programs Biomed 53:15-25

27. Yoo TS, Ackerman MJ, Lorensen WE, Schroeder W, Chalana V, Aylward S, Metaxes D, Whitaker R (2002) Engineering and algorithm design for an image processing API: a technical report on ITK - the insight toolkit. IOS Press, Amsterdam, pp 586-592

28. Collins DL, Neelin P, Peters TM, Evans AC (1994) Automatic 3D intersubject registration of MR volumetric data in standardized Talairach space. J Comput Assist Tomogr 18:192-205

29. Papademetris X, Jackowski N, Rajeevan N, Constable RT, Staib LH. BioImage Suite: an integrated medical image analysis suite, Section of Bioimaging Sciences, Dept. of Diagnostic Radiology, Yale School of Medicine. http://www.bioimagesuite.org

30. Studholme C, Hill DLG, Hawkes DJ (1999) An overlap invariant entropy measure of 3D medical image alignment. Pattern Recogn 32:71-86

31. Scheltens P, Leys D, Barkhof F, Huglo D, Weinstein HC, Vermersch P, Kuiper M, Steinling M, Wolters EC, Valk J (1992) Atrophy of medial temporal lobes on MRI in 'probable' Alzheimer's disease and normal ageing: diagnostic value and neuropsychological correlates. J Neurol Neurosurg Psychiatry 55 (10):967-972

32. Bresciani L, Rossi R, Testa C, Geroldi C, Galluzzi S, Laakso MP, Beltramello A, Soininen H, Frisoni GB (2005) Visual assessment of medial temporal atrophy on MR films in Alzheimer's disease: comparison with volumetry. Aging Clin Exp Res 17:8-13

33. Gao FQ, Black SE, Leibovitch FS, Callen DJ, Rockel CP, Szalai JP (2004) Linear width of the medial temporal lobe can discriminate Alzheimer's disease from normal aging: the Sunnybrook dementia study. Neurobiol Aging 25:441-448

34. Davies RR, Scahill VL, Graham A, Williams GB, Graham KS, Hodges JR (2009) Development of an MRI rating scale for multiple brain regions: comparison with volumetrics and with voxel-based morphometry. Neuroradiology 51:491-503

35. Jack CR Jr, Petersen RC, O'Brien PC, Tangalos EG (1992) MRbased hippocampal volumetry in the diagnosis of Alzheimer's disease. Neurol 42:183-188 
36. Chan D, Fox NC, Scahill RI, Crum WR, Whitwell JL, Leschziner G, Rossor AM, Stevens JM, Cipolotti L, Rossor MN (2001) Patterns of temporal lobe atrophy in semantic dementia and Alzheimer's disease. Ann Neurol 49:433-442

37. Barnes J, Whitwell JL, Frost C, Josephs KA, Rossor M, Fox NC (2006) Measurements of the amygdala and hippocampus in pathologically confirmed Alzheimer disease and frontotemporal lobar degeneration. Arch Neurol 63:1434-1439
38. Lerch JP, Pruessner J, Zijdenbos AP, Collins DL, Teipel SJ, Hampel H, Evans AC (2008) Automated cortical thickness measurements from MRI can accurately separate Alzheimer's patients from normal elderly controls. Neurobiol Aging 29:23-30 39. Wald NJ, Murphy P, Major P, Parkes C, Townsend J, Frost C (1995) UKCCCR multicentre randomised controlled trial of one and two view mammography in breast cancer screening. BMJ 311:1189-1193 